\title{
Erratum
}

\section{Erratum: Jogan and Stocker, Signal Integration in Human Visual Speed Perception}

In the article "Signal Integration in Human Visual Speed Perception" by Matjaž Jogan and Alan A. Stocker, which appeared on pages 9381-9390 of the June 24, 2015 issue, two errors were discovered and are corrected below.

1. In Materials and Methods, Stimulus calibration, second column, first paragraph on page 9382, the equation should read as follows:

This slope value is equivalent to a stimulus noise level of $\sigma=0.6 / \sqrt{2}$ according to signal detection theory (SDT; Green and Swets, 1966).

2. In the References, first column, second reference on page 9389, the names and the citation should read:

Bayes Mr., Price Mr. (1763) An essay toward solving a problem in the doctrine of chances. By the late Rev. Mr. Bayes, FRS communicated by Mr. Price, in a letter to John Canton, AMFRS. Philosophical Transactions (1683-1775), 370-418.

These corrections do not affect the conclusions and interpretations of the paper. The equation and reference have been corrected on the online PDF version.

DOI:10.1523/JNEUROSCI.2956-15.2015 